\title{
Chemotaxonomic Markers in Essential Oils of Hypenia (Mart. ex Benth.) R. Harley
}

\author{
Maria T. Faria ${ }^{a, b}$ Deomar P. Costa, ${ }^{c}$ Eliane C. Vilela, ${ }^{c}$ Dalva G. Ribeiro, ${ }^{a}$ \\ Heleno D. Ferreira, ${ }^{b}$ Suzana C. Santos, ${ }^{c}$ José C. Seraphin $^{d}$ and Pedro H. Ferri*, \\ ${ }^{a}$ Instituto de Biologia, Universidade de Brasília, CP 4451, 70910-900 Brasília-DF, Brazil \\ ${ }^{b}$ Instituto de Ciências Biológicas, 'Instituto de Química and 'Instituto de Matemática e Estatística, \\ Universidade Federal de Goiás, CP 131, 74001-970 Goiânia-GO, Brazil
}

\begin{abstract}
O Cerrado Central brasileiro é considerado o centro de origem e dispersão de Hypenia (Lamiaceae: subtribo Hyptidineae) na América do Sul. O gênero tem sido mantido sem divisão, ou subdividido em duas ou cinco seções taxonômicas, baseadas quase exclusivamente a partir de um conjunto limitado de características florais. Neste trabalho, uma delimitação química interespecífica é descrita a fim de complementar as análises das características botânicas. Análise multivariada dos óleos essenciais de dez espécies na fase vegetativa suporta a divisão taxonômica do gênero em duas seções. Grupo I incluiu quatro espécies da seção Laxiflorae em adição à H. irregularis, os quais foram caracterizados pelos mais altos percentuais de espatulenol e óxido de cariofileno. Grupo II conteve cinco espécies da seção Densiflorae com $\alpha$-cadinol, $\alpha$-muuroleno e $\alpha$-muurolol como constituintes principais. Cadinanos e germacranos/aromadendranos parecem ser as classes marcadoras quimiotaxonômicas nas seções Densiflorae e Laxiflorae, respectivamente. Além disso, análise de redundância canônica não indicou correlação significante entre fatores edáficos com quimiovariação nos óleos essenciais, sugerindo que em Hypenia os óleos são constituídos por substâncias constitutivas e não induzidas pelo ambiente.
\end{abstract}

Central Brazilian Cerrado is regarded as the center of origin and dispersion of Hypenia (Lamiaceae: subtribe Hyptidineae) in South America. The genus has either remained undivided or been divided into two or seven taxonomic sections, based almost exclusively on a limited range of floral features. Interspecific chemical delimitation to complement analyses of botanical traits is described. Multivariate analysis of essential oils of ten species in the vegetative stage supports the taxonomic division in two sections. Cluster I included four species belonging to section Laxiflorae in addition to $H$. irregularis, which were characterized by the highest percentages of spathulenol and caryophyllene oxide. Cluster II contained five species belonging to section Densiflorae with $\alpha$-cadinol, $\alpha$-muurolene and $\alpha$-muurolol as major constituents. Cadinanes and germacranes/ aromadendranes seem to be chemotaxonomic markers of sections Densiflorae and Laxiflorae, respectively. Furthermore, redundancy analysis revealed no significant correlation between edaphic factors and oil chemovariations, suggesting that Hypenia oils consist of constitutive rather than induced compounds.

Keywords: Hypenia, essential oil, chemical variability, chemotaxonomy, multivariate analysis

\section{Introduction}

In Cerrado areas throughout Brazil and parts of Eastern South America, the recently redefined and amplified Lamiaceae family is mainly represented by the neotropical subtribe Hyptidinae, tribe Ocimeae, whose patterns of floristic and taxonomic variation have resulted in endemic genera and a large number of new species. ${ }^{1-3}$ Furthermore,

*e-mail: pedro@quimica.ufg.br it is characterized by sternotribic flowers whose stamens are held in the compressed lower lip of the corolla, forming an explosive pollination mechanism., ${ }^{4,5}$

Nine genera of the Hyptidinae have been identified, apparently in two separate clades: one represented by Eriope Humb. \& Bonpl. ex Benth., Hypenia (Mart. ex Benth.) R. Harley and Eriopidion (Harley) and the other represented by Hyptis Jacq., together with Peltodon Pohl, Marsypianthes Mart. ex Benth., Rhaphiodon Schau., the only one restricted to Central America, 
Asterohyptis Epling and the genus Hyptidendron Harley. ${ }^{1,3}$ Hypenia was separated from Hyptis section Hypenia based largely on the number of chromosomes, pollen surface and stem anatomy, as well as by traditional characters. ${ }^{1,4}$ In addition, it represents a monophyletic group based on nuclear and plastid DNA. ${ }^{3}$

Its twenty seven recognized species have remained taxonomically undivided or have been distributed into sections Densiflorae Benth. and Laxiflorae Benth., based on lax or dense inflorescence. ${ }^{4,6}$ Although Briquet ${ }^{7}$ initially proposed seven sections, some species have now been assigned to the genus Eriope. ${ }^{5}$ They are usually found in oligotrophic and sandy soils with high aluminum contents and low nutrient availability, and are spread over some regions of Venezuela, Bolivia, Paraguay and Southern Brazil. A new and unique species representative of the genus Hypenia in Mexico has recently been described. ${ }^{8}$ In Brazil, they are more common in Cerrado regions which hold greater diversity and endemism. ${ }^{1,4}$ Similarly to the correlated genus Hyptis, Hypenia species are aromatic and are frequently cited in Brazilian Cerrado for their ethnobotanical use, such as the infusion or decoction of leaves in the treatment of flu, common cold and other respiratory diseases.. ${ }^{9}$ Moderate radical scavenging and antioxidant activities of methanol extracts of leaves and stems of $H$. salzmannii (Benth.) R. Harley have also been reported. ${ }^{10}$

The botanical keys of the two Hypenia sections show that the characters used for their distinction derived almost exclusively from a limited range of floral features. ${ }^{6}$ These difficulties may be partly attributed to probable hybridization between sympatric species, as in Eriope, in addition to the small number of specimens deposited in a herbarium. ${ }^{5}$ For example, H. paradisi were, until recently, collected in just two field trips. ${ }^{411,12}$ Since all of them are morphologically and anatomically similar, it is important to find alternative methods of interspecific chemical identification in order to complement floral trait analyses.

Recent studies described the chemical constituents of essential oils from thirteen Brazilian flowering Hypenia species, as well as compared the chemotaxonomic significance of essential oil chemovariations with botanical taxonomy by means of multivariate analysis, to determine taxa distribution patterns into two taxonomical sections. ${ }^{12}$

The results obtained for the chemical composition of essential oils of ten Hypenia taxa are now reported, including four new species, all of which were located in Central Brazilian Cerrado in the vegetative stage. Intraspecific variability was also verified by processing more than one population per taxon for three species.

\section{Results and Discussion}

The Central Brazilian Cerrado region is regarded as the center of origin and dispersion of Hypenia in South America. ${ }^{5}$ Despite the great diversity of species that live in these areas, essential oil composition is only known from flowering samples. ${ }^{12}$ The lack of phenophase influence on chemovariation may be partly attributed to the small number of specimens from wild populations and which are deposited in the herbarium. ${ }^{13}$ In addition, all of the Hypenia species are morphologically similar and floral traits are very important for their botanical identification. ${ }^{4,6}$ In our study, Hypenia species were collected from distinct physiognomic sites in which the soil features are representative of the Cerrado fertility gradient. ${ }^{14}$ The main soil features that showed the highest variations for the species under investigation were clay, silt, $\mathrm{pH}, \mathrm{Al}^{3+}$, cationic exchange concentration (CEC), organic matter and macronutrients $\left(\mathrm{K}^{+}, \mathrm{Ca}^{2+}, \mathrm{Mg}^{2+}, \mathrm{P}\right)$.

Essential oil compositions were obtained from ten species in the vegetative stage, six of which belonged to section Densiflorae (H. chapadensis (Hcha), H. inelegans (Hine), H. irregularis (Hirr), H. jorgeana (Hjor), $H$. paradisi (Hpar) and H. pruinosa (Hpru)) and four of which belonged to section Laxiflorae (H. calycina (Hcal), H. macrantha (Hmac), H. puchra (Hpuc) and H. rupestre (Hrup)), following Epling's classification. ${ }^{6}$ As regards plant collection, $H$. chapadensis, $H$. jorgeana, $H$. puchra and $H$. rupestre are currently undescribed species in the genus. A list of the taxa investigated, as well as their provenance and vouchers, are shown in the Supplementary Information (SI) section (Table S1).

Unlike their rich essential oil allies, such as Hyptis, ${ }^{5}$ vegetative Hypenia species showed low essential oil yields $(0.04 \pm 0.02 \%)$ and failed to show significant differences in relation to flowering species, ${ }^{12}$ which suggests that Hypenia may be an oil-poor genus. A total of eighty five oil constituents was identified (Table 1) and nearly half presented an average $>0.5 \%$, accounting for $86-97 \%$ of the sampled data.

Essential oil compositions revealed a predominance of sesquiterpenes (43.9-100\%), particularly high contents of sesquiterpene hydrocarbons in most species (23.4-83.9\%). Oxygenated sesquiterpenes were the majority only in $H$. irregularis (Hirr, $74.7 \%$ ). With the exception of population Hprul of $H$. pruinosa, which showed significant levels of aromatic compounds and esters (other constituents; $53.1 \%$ ), all the other samples had lower levels of such compounds $(<10 \%)$.

Essential oil compositions of all Hypenia species contained $\alpha$-copaene (range of $0.74-12.5 \%$, average value of $3.20 \%)$ and (E)-caryophyllene (1.99-27.2\%, 
Table 1. Percentages of essential oil constituents from Hypenia species ${ }^{\mathrm{b}}$ according to Epling's classifications

\begin{tabular}{|c|c|c|c|c|c|c|c|c|c|c|c|c|c|c|c|c|}
\hline & \multirow{2}{*}{ Constituent } & \multirow{2}{*}{$\mathrm{RI}^{\mathrm{a}}$} & \multicolumn{5}{|c|}{ Laxiflorae section } & \multicolumn{9}{|c|}{ Densiflorae section } \\
\hline & & & Hcal1 & Hcal2 & $\mathrm{Hmac}$ & $\mathrm{Hpuc}^{\mathrm{d}}$ & Hrup $^{\mathrm{d}}$ & Hcha $^{\mathrm{d}}$ & Hine & Hirr & 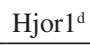 & Hjor $^{\mathrm{d}}$ & Hpar & Hpru1 & Hpru2 & Hpru3 \\
\hline 1 & Heptanal & 897 & - & - & - & - & - & - & - & - & 0.52 & - & - & - & 0.37 & - \\
\hline 2 & Tricyclene & 923 & - & - & - & 0.48 & - & - & - & - & - & - & - & - & - & - \\
\hline 3 & $\alpha$-Pinene & 929 & - & - & - & 1.51 & - & - & - & - & - & - & - & 0.49 & - & - \\
\hline 4 & (2E)-Heptenal & 949 & - & - & - & - & - & - & - & - & 0.34 & - & - & - & 1.09 & - \\
\hline 5 & $\beta$-Pinene & 972 & - & 1.80 & - & 3.18 & - & - & - & - & - & - & - & 0.58 & - & - \\
\hline 6 & Myrcene & 986 & - & - & - & 0.78 & - & - & - & - & - & - & - & - & - & - \\
\hline 7 & $\rho$-Cymene & 1020 & - & - & - & 2.33 & - & 8.74 & - & 0.76 & 1.87 & 2.18 & 1.42 & - & 1.63 & - \\
\hline 8 & Limonene & 1024 & - & - & - & - & - & 0.82 & - & - & - & - & - & - & 10.3 & - \\
\hline 9 & $\beta$-Phellandrene & 1025 & - & 2.24 & - & 4.41 & - & - & - & - & - & - & - & - & - & - \\
\hline 10 & 1,8-Cineole & 1028 & - & - & - & 6.50 & - & - & - & - & 0.93 & 0.96 & - & - & - & - \\
\hline 11 & $\gamma$-Terpinene & 1054 & - & - & - & 2.58 & - & - & - & - & - & - & - & - & - & - \\
\hline 12 & Acetophenone $^{c}$ & 1063 & - & - & - & - & - & - & - & - & - & - & - & 21.4 & - & - \\
\hline 13 & Linalool & 1096 & - & - & 0.35 & 1.77 & - & 0.52 & - & 1.10 & - & - & - & 1.14 & - & 0.48 \\
\hline 14 & trans-Thujone & 1113 & - & - & - & - & - & 1.49 & - & - & - & - & - & - & - & - \\
\hline 15 & (2E)-Nonen-1-al & 1155 & - & - & - & - & - & - & - & - & 0.66 & - & - & - & - & - \\
\hline 16 & $\alpha$-Copaene ${ }^{c}$ & 1374 & 1.86 & 12.5 & 0.99 & 1.14 & 4.38 & 3.01 & 1.66 & 1.13 & 2.48 & 2.90 & 0.74 & 1.75 & 1.66 & 8.61 \\
\hline 17 & Geranyl acetate & 1381 & 1.13 & - & - & - & - & - & - & - & - & - & - & - & - & - \\
\hline 18 & $\beta$-Bourbonene & 1382 & 2.33 & 2.08 & 2.01 & - & 2.52 & 2.06 & 1.45 & 1.39 & 1.29 & 1.50 & 2.17 & 1.20 & 1.72 & 1.37 \\
\hline 19 & $\beta$-Elemene & 1389 & 1.48 & - & 1.17 & - & 2.44 & 3.72 & - & - & - & - & - & 1.11 & - & - \\
\hline 20 & Methyl eugenol & 1400 & - & - & - & - & - & - & - & - & - & - & - & 0.91 & - & - \\
\hline 21 & Longifolene & 1407 & - & - & - & - & - & - & - & - & - & - & - & 0.64 & - & - \\
\hline 22 & $\alpha$-Gurjunene & 1412 & - & - & - & 0.66 & - & - & - & - & - & - & - & - & - & - \\
\hline 23 & $\beta$-Funebrene & 1415 & - & - & - & - & - & - & - & - & 1.36 & 1.64 & - & - & - & - \\
\hline 24 & $(E)$-Caryophyllene ${ }^{\mathrm{c}}$ & 1417 & 5.39 & 27.2 & 4.33 & 12.4 & 8.07 & 3.88 & 9.01 & 3.71 & 3.69 & 4.45 & 3.81 & 1.99 & 2.01 & 4.36 \\
\hline 25 & $\beta$-Copaene & 1427 & - & - & 0.37 & - & - & - & 0.66 & - & - & - & - & - & - & - \\
\hline 26 & $\alpha$-trans-Bergamotene & 1434 & - & - & - & - & - & - & 0.66 & - & - & - & - & 1.39 & - & 1.74 \\
\hline 27 & $\alpha$-Guaiene & 1436 & - & - & - & - & - & - & - & - & - & - & - & 0.58 & - & - \\
\hline 28 & Aromadendrene & 1437 & - & - & - & 1.65 & - & - & - & - & - & - & - & - & - & - \\
\hline 29 & Neryl propanoate ${ }^{c}$ & 1450 & - & - & - & - & - & - & - & - & - & - & - & 12.8 & - & - \\
\hline 30 & $\alpha$-Humulene & 1451 & 1.64 & 2.35 & 1.15 & 1.34 & 1.20 & 2.47 & 6.13 & 1.03 & 1.35 & 1.63 & 2.67 & - & - & 3.07 \\
\hline 31 & Geranyl acetone ${ }^{c}$ & 1451 & - & - & - & - & - & - & - & - & 2.34 & 2.81 & 1.70 & - & 1.60 & 3.62 \\
\hline 32 & allo-Aromadendrene & 1458 & - & - & - & - & - & - & - & - & - & - & - & - & - & 3.89 \\
\hline 33 & trans-Cadina-1(6),4-diene ${ }^{c}$ & 1474 & - & - & - & - & - & - & 2.77 & 1.49 & 3.70 & 2.99 & 2.74 & 1.55 & - & - \\
\hline 34 & $\gamma$-Gurjunene & 1474 & - & - & - & - & - & 1.88 & - & - & - & - & - & - & 3.84 & 0.73 \\
\hline 35 & $\gamma$-Muurolene ${ }^{c}$ & 1479 & 18.9 & - & 33.8 & 0.72 & - & 4.08 & 12.5 & 5.91 & 7.71 & 8.71 & 23.5 & 2.39 & 6.17 & 7.20 \\
\hline 36 & $\gamma$-Himachalene & 1484 & - & - & 0.31 & - & - & - & 0.59 & - & 1.39 & 1.50 & 1.10 & - & - & - \\
\hline 37 & Germacrene $\mathrm{D}^{\mathrm{c}}$ & 1484 & - & 27.2 & - & - & 38.7 & - & - & - & - & - & - & - & - & - \\
\hline 38 & $\beta$-Selinene ${ }^{c}$ & 1484 & - & 2.78 & - & - & - & 2.12 & - & - & - & - & - & 2.65 & 5.07 & 2.13 \\
\hline 39 & $\delta$-Selinene ${ }^{c}$ & 1493 & - & - & - & 18.2 & - & 3.04 & - & - & - & - & - & 3.65 & 6.23 & 5.81 \\
\hline 40 & Indipone & 1493 & - & - & - & - & - & - & - & - & 3.56 & 3.44 & - & - & - & - \\
\hline 41 & Bicyclogermacrene $^{c}$ & 1495 & 10.3 & 1.80 & 19.6 & - & 6.55 & - & 6.56 & 4.66 & 3.08 & 3.62 & 9.06 & - & - & - \\
\hline 42 & $\alpha$-Muurolene ${ }^{c}$ & 1497 & - & - & - & - & 0.74 & 4.00 & 0.95 & - & 7.80 & 9.33 & 3.68 & 1.94 & 5.32 & 2.21 \\
\hline 43 & Germacrene A & 1503 & - & - & 0.90 & - & 0.86 & 2.80 & - & - & - & - & - & 1.06 & - & - \\
\hline 44 & $\gamma$-Cadinene ${ }^{c}$ & 1511 & 1.21 & 1.74 & 0.98 & 0.67 & - & 1.34 & 1.97 & 1.10 & 2.96 & 3.14 & 3.71 & 2.19 & 4.35 & 1.62 \\
\hline 45 & trans-Cycloisolongifol-5-ol & 1514 & - & - & - & 5.37 & - & - & - & - & - & - & - & - & - & - \\
\hline 46 & Cubebol & 1514 & - & - & - & - & 2.16 & - & 0.50 & - & - & - & - & - & - & - \\
\hline 47 & trans-Calamenene $\mathrm{e}^{\mathrm{c}}$ & 1520 & - & - & - & - & - & 2.29 & - & - & 4.96 & 5.58 & - & - & 3.42 & - \\
\hline 48 & $\delta$-Cadinene ${ }^{c}$ & 1521 & 2.18 & 4.39 & 1.49 & 1.50 & 5.27 & - & 4.73 & 3.00 & - & - & 4.26 & 5.96 & - & 7.58 \\
\hline
\end{tabular}


Table 1. continuation

\begin{tabular}{|c|c|c|c|c|c|c|c|c|c|c|c|c|c|c|c|c|}
\hline & \multirow{2}{*}{ Constituent } & \multirow{2}{*}{$\mathrm{RI}^{\mathrm{a}}$} & \multicolumn{5}{|c|}{ Laxiflorae section } & \multicolumn{9}{|c|}{ Densiflorae section } \\
\hline & & & Hcal1 & Hcal2 & Hmac & $\mathrm{Hpuc}^{\mathrm{d}}$ & Hrup $^{d}$ & $\mathrm{Hcha}^{\mathrm{d}}$ & Hine & Hirr & 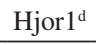 & Hjor $^{\mathrm{d}}$ & Hpar & Hpru1 & Hpru2 & Hpru3 \\
\hline 49 & Zonarene & 1524 & - & - & - & - & - & - & - & - & - & - & - & 0.36 & - & 0.50 \\
\hline 50 & $\alpha$-Cadinene & 1535 & - & - & - & - & - & - & - & - & - & - & 1.08 & - & - & - \\
\hline 51 & $\alpha$-Calacorene $e^{c}$ & 1539 & - & - & - & - & - & 1.84 & - & - & 3.73 & 3.79 & 2.76 & - & 2.60 & - \\
\hline 52 & Italicene epoxide & 1550 & 0.67 & - & - & - & - & - & - & - & - & - & - & - & - & - \\
\hline 53 & Germacrene B & 1555 & 0.71 & 1.81 & 0.58 & - & - & 0.99 & 10.3 & - & - & - & - & - & - & - \\
\hline 54 & Caryophyllenyl alcohol & 1568 & - & - & - & - & - & 1.26 & - & - & - & - & - & - & - & - \\
\hline 55 & Caryolan-8-ol ${ }^{\mathrm{c}}$ & 1569 & - & - & - & - & - & - & - & - & 5.00 & 4.72 & - & - & - & - \\
\hline 56 & Spathulenol $^{c}$ & 1581 & 30.4 & 3.50 & 11.1 & 9.56 & 6.25 & 3.13 & 7.63 & 47.8 & 3.21 & 3.33 & 3.79 & - & 4.07 & 6.61 \\
\hline 57 & trans-Sesquisabinene hydrate & 1581 & - & - & - & - & - & 4.85 & - & - & 1.73 & - & - & 2.77 & 1.81 & - \\
\hline 58 & Caryophyllene oxide ${ }^{c}$ & 1584 & 12.5 & 6.29 & 4.07 & 6.28 & 7.44 & 1.37 & 5.19 & 13.4 & 2.13 & 4.18 & 4.13 & - & 6.04 & 3.88 \\
\hline 59 & Globulol $^{\mathrm{c}}$ & 1589 & - & - & 1.87 & 10.1 & 5.20 & - & - & - & 1.42 & - & - & 0.68 & - & 3.32 \\
\hline 60 & (E)-Dihydroapofarnesol & 1590 & - & - & - & - & - & - & - & - & - & - & - & - & 7.80 & - \\
\hline 61 & Guaiol & 1600 & - & - & - & - & - & - & - & - & 1.17 & - & - & - & - & - \\
\hline 62 & Ledol & 1601 & - & - & - & - & - & - & - & - & - & - & - & - & - & 1.84 \\
\hline 63 & Humulene epoxide & 1608 & 0.86 & - & 0.90 & - & - & 1.86 & 2.62 & 1.51 & 1.05 & - & - & - & - & 0.98 \\
\hline 64 & Junenol & 1616 & - & - & - & - & - & - & 2.09 & - & - & - & - & - & - & - \\
\hline 65 & $\alpha$-Corocalene & 1621 & - & - & - & - & - & - & - & - & 0.91 & - & - & - & - & - \\
\hline 66 & 1-epi-Cubenol ${ }^{\mathrm{c}}$ & 1627 & - & - & - & 0.41 & - & 1.08 & - & 1.45 & 3.96 & 2.49 & 1.54 & 0.89 & 2.46 & 3.63 \\
\hline 67 & Muurola-4,10(14)-dien-1 $\beta$-ol & 1627 & - & - & - & - & - & - & 4.98 & - & - & - & - & - & - & - \\
\hline 68 & $\gamma$-Eudesmol & 1631 & - & - & - & - & - & - & - & - & 0.62 & - & - & - & - & - \\
\hline 69 & epi- $\alpha$-Cadinol & 1637 & - & - & - & 1.28 & - & - & - & - & - & - & 8.90 & 2.88 & - & 7.27 \\
\hline 70 & allo-Aromadendrene epoxide & 1637 & - & - & - & - & - & - & - & 1.06 & - & - & - & - & - & - \\
\hline 71 & Selina-3,11-dien-6 $\alpha$-ol & 1638 & - & - & 1.06 & - & - & - & 1.01 & - & - & - & - & - & - & - \\
\hline 72 & $\alpha$-Muurolol ${ }^{\mathrm{c}}$ & 1641 & - & - & 1.98 & - & - & 4.45 & 3.72 & 1.66 & 8.88 & 10.5 & - & - & 6.71 & - \\
\hline 73 & Cubenol $^{c}$ & 1645 & - & - & 0.77 & - & 0.99 & 2.25 & 1.48 & 1.17 & 3.68 & 4.40 & 2.59 & - & 2.24 & 2.11 \\
\hline 74 & Pogostol & 1654 & - & - & 8.80 & - & - & - & - & - & - & - & - & - & - & - \\
\hline 75 & $\alpha-$ Cadinol $^{\mathrm{c}}$ & 1654 & 3.27 & - & - & 1.47 & - & - & 8.07 & 3.18 & 4.34 & 5.92 & 11.2 & 6.28 & 7.09 & 7.37 \\
\hline 76 & Selin-11-en- $4 \alpha$-ol & 1660 & - & 2.31 & - & - & 4.53 & 27.1 & - & - & - & - & 0.37 & - & - & - \\
\hline 77 & cis-Calamenen-10-ol & 1661 & - & - & - & - & - & - & - & - & 0.55 & - & - & - & - & - \\
\hline 78 & $\begin{array}{l}\text { 14-Hydroxy-9-epi-(E)- } \\
\text { caryophyllene }\end{array}$ & 1670 & 1.41 & - & - & 0.56 & - & - & - & 1.46 & - & - & - & - & - & - \\
\hline 79 & Cadalene & 1672 & - & - & - & - & - & 0.84 & - & - & 2.81 & 2.70 & 1.92 & - & 2.32 & - \\
\hline 80 & Mustakone $^{\mathrm{c}}$ & 1676 & 2.25 & - & - & - & - & - & - & - & - & - & - & - & - & - \\
\hline 81 & Eudesma-4(15),7-dien-1 $\beta$-ol ${ }^{\mathrm{c}}$ & 1685 & 1.54 & - & 1.46 & - & 2.69 & - & 1.22 & 1.97 & - & - & - & - & - & - \\
\hline 82 & Eudesm-7(11)-en-4-ol & 1694 & - & - & - & - & - & 0.68 & 0.68 & - & - & - & - & - & - & - \\
\hline 83 & Benzyl benzoate & 1759 & - & - & - & - & - & - & - & - & - & - & - & 0.60 & - & - \\
\hline 84 & Hexahydrofarnesyl acetone ${ }^{c}$ & 1843 & - & - & - & - & - & - & - & - & - & 1.61 & - & 9.58 & - & 2.72 \\
\hline 85 & $(5 E, 9 E)$-Farnesyl acetone ${ }^{c}$ & 1915 & - & - & - & - & - & - & - & - & - & - & - & 7.87 & - & 3.45 \\
\hline & Monoterpene hydrocarbons & & - & 4.04 & - & 12.7 & - & 9.56 & - & 0.76 & 1.87 & 2.18 & 1.42 & 1.07 & 11.94 & - \\
\hline & Oxygenated monoterpenes & & - & - & 0.35 & 10.9 & - & 2.01 & - & 1.10 & 0.93 & 0.96 & - & 1.14 & - & 0.48 \\
\hline & Sesquiterpene hydrocarbons & & 46.0 & 83.9 & 67.7 & 38.3 & 72.9 & 40.4 & 60.3 & 23.4 & 52.8 & 56.9 & 63.2 & 30.4 & 44.7 & 50.8 \\
\hline & Oxygenated sesquiterpenes & & 52.9 & 12.1 & 32.0 & 35.0 & 27.1 & 48.1 & 38.7 & 74.7 & 37.7 & 35.5 & 32.6 & 13.5 & 38.2 & 37.0 \\
\hline & Others & & 1.13 & - & - & - & - & - & - & - & 3.86 & 4.42 & 1.70 & 53.1 & 3.06 & 9.8 \\
\hline & Oil yield / \%, m/m & & 0.04 & 0.04 & 0.03 & 0.06 & 0.05 & 0.06 & 0.03 & 0.02 & 0.04 & 0.03 & 0.05 & 0.05 & 0.09 & 0.03 \\
\hline & Total identified & & 99.9 & 99.9 & 100 & 90.9 & 100 & 99.9 & 99.0 & 99.9 & 96.3 & 100 & 98.8 & 98.2 & 96.5 & 98.1 \\
\hline
\end{tabular}


average value of $6.74 \%)$. The most abundant constituents were spathulenol $(0-47.8 \%, 10.1 \%)$, caryophyllene oxide $(0-13.4 \%, 5.50 \%)$ and germacrene D $(0-38.7 \%$, $4.71 \%$ ), which showed high values in species from section Laxiflorae with the addition of $H$. irregularis. However, $\alpha$-cadinol $(0-11.2 \%, 4.16 \%)$ and $\alpha$-muurolene $(0.95-9.33 \%$, $2.57 \%)$ were the main constituents in species from section Densiflorae with the exception of $H$. irregularis. $\gamma$-Muurolene $(0-33.8 \%, 9.40 \%),(E)$-caryophyllene and bicyclogermacrene $(0-19.6 \%, 4.66 \%)$ showed high contents in both botanical sections. These results are in agreement with previous studies as regards flowering Hypenia essential oils, ${ }^{12}$ although they differ in the low content of selin-11-en- $4 \alpha$-ol (vegetative samples: $0-27.1 \%, 2.45 \%$; flowering samples: $0-34.8 \%, 9.76 \%)$ and a variety of minor constituents. High contents of $(E)$-caryophyllene and derivatives in Hypenia, which are known to prevent herbivory regardless of the developmental stages in question, ${ }^{15}$ suggest that these essential oil constituents may provide Hypenia with selective advantages over native species in terms of habitat adaptation and consequently of high endemism.

Despite the fact that the sampling sites showed a relatively significant difference as regards soil chemical composition and texture, canonical redundancy analysis (RDA) revealed no correlation between edaphic variables and essential oil chemovariations. A significance test with an unrestricted Monte Carlo permutation technique (999 permutations) found Fischer's $F$-ratio for the first canonical axis $(F$-value $=1.087 ; p=0.439)$ and for all canonical axes $(F=1.040 ; p=0.394)$, giving signs that patterns did arise by chance. ${ }^{16} \mathrm{~A}$ strong but non-significant interset correlation $(R=0.995$; first RDA axis, $p=0.085$; trace statistics, $p=0.054$ ) was observed between clay, silt, $\mathrm{Al}^{3+}, \mathrm{Mn}^{2+}$ and $\mathrm{Zn}^{2+}$ contents and H. calycina, $H$. jorgeana and $H$. pruinosa collected at different sampling sites. These results suggest that Hypenia oils were genetically rather than environmentally influenced, in agreement with previous studies that reported genetic control in essential oil variations of flowering Hypenia species. ${ }^{12}$ Therefore, essential oil chemovariations in the vegetative stage may also contribute to chemotaxonomic or phylogenetic relationships within the genus. Similar results were described for species of Hypericum L. (Hypericaceae) from different locations and phenophases. Even though no major differences were observed in essential oil compositions within each species, the range of dominant constituents supports the sectional separation of species. ${ }^{17}$

To assess the use of essential oil constituents in identifying taxonomic relationships among species, multivariate analysis by principal components analysis (PCA) and nearest neighbor complete linkage cluster analysis were initially performed with oil constituent levels $\geq 0.5 \%$ ( 14 samples $\times 41$ variables $=574$ pieces of data). After the variable modeling which aimed to stabilize the hierarchical structure (final matrix, $14 \times 28=392$ ), the relative position of the PCA based taxa is shown in Figure 1. The first PC accounts for $35.0 \%$ of total variance and separates populations of H. calycina (Hcal1 and Hcal2), H. irregularis (Hirr), H. macrantha (Hmac), H. puchra (Hpuc) and H. rupestre (Hrup) above the 97\% confidence level from other specimens according to the highest contents of spathulenol, germacrene $\mathrm{D},(E)$-caryophyllene and caryophyllene oxide. The second PC (19.3\% of total variation) distinguishes $(p<0.005) H$. pruinosa populations (Hpru1, Hpru2 and Hpru3) mainly because of the highest contents of neryl propanoate and $\alpha$-cadinol.

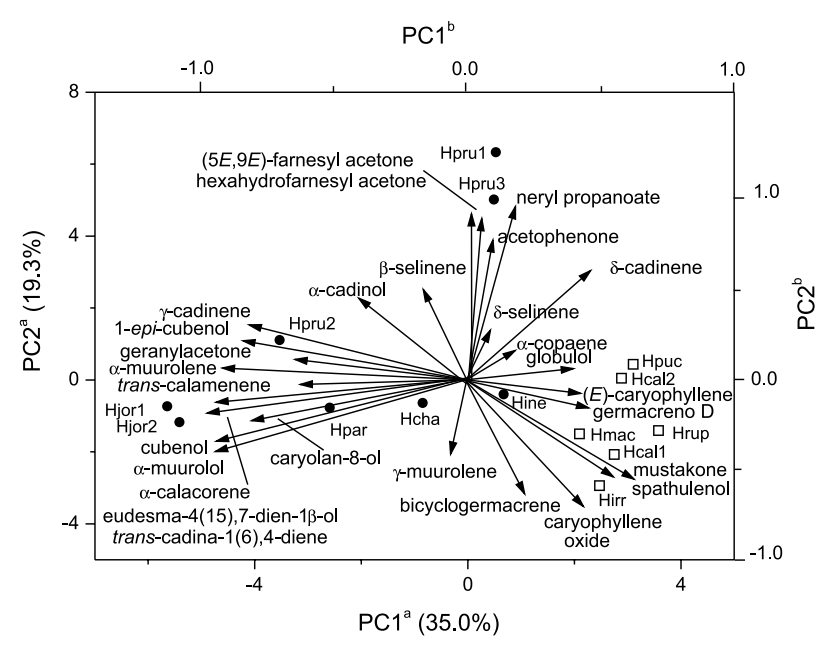

Figure 1. PCA biplot of Hypenia species essential oil, according to the clusters they belong to: I ( $\square)$; II $(\bullet) .{ }^{a}$ Axes refer to scores from the samples. ${ }^{\mathrm{b}}$ Axes refer to loadings from oil constituents represented as vectors from the origin. Values between parentheses refer to the explained variance on each principal component. Hypenia samples: Hcal $=H$. calycina; Hcha $=H$. chapadensis $;$ Hine $=H$. inelegans; Hirr $=H$. irregularis; Hjor $=H$. jorgeana $;$ Hmac $=H$. macrantha $;$ Hpar $=H$. paradisi $;$ $\mathrm{Hpru}=$ H. pruinosa $;$ Hpuc $=$ H. puchra $;$ Hrup $=$ H. rupestre.

Therefore, two types of essential oils were identified. Cluster I revealed four species of the section Laxiflorae, in addition to $H$. irregularis, which were characterized $(p<0.009)$ by the highest percentages of spathulenol $(18.1 \pm 17.4 \%),(E)$-caryophyllene $(10.2 \pm 8.90 \%)$ and caryophyllene oxide $(8.34 \pm 3.76 \%)$. Cluster II revealed five species of the section Densiflorae containing $\alpha$-cadinol $(6.29 \pm 3.23 \%), \alpha$-muurolene $(4.40 \pm 2.93 \%), \alpha$-muurolol $(4.28 \pm 4.15 \%)$ and $\gamma$-cadinene $(2.66 \pm 1.06 \%)$ as the main constituents. Figure 2 shows the similarities between taxa in terms of Euclidean distances, all of which originated from the cluster analysis via PC scores.

A similar hierarchical structure emerges when it is considered the oil constituents grouped together according 


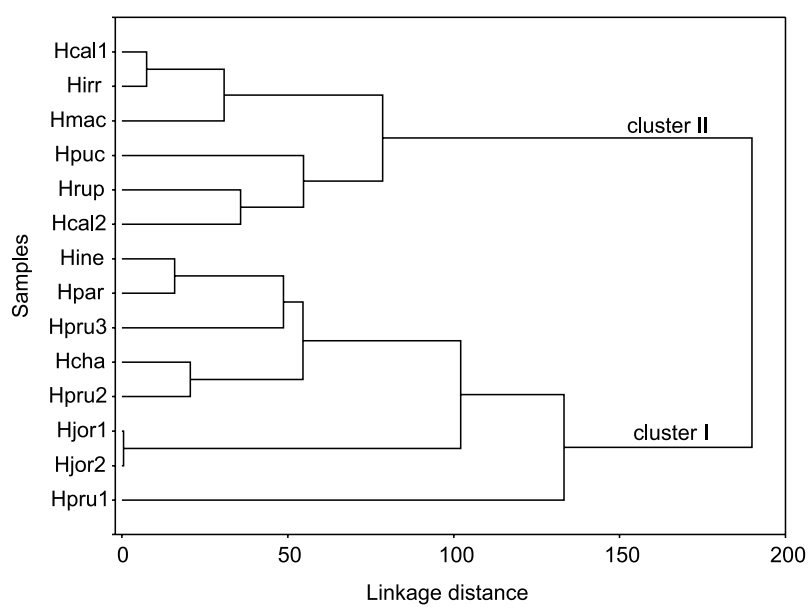

Figure 2. Dendrogram representing similarity relationships among Hypenia species based on essential oil constituents, according to the clusters they belong to: I and II. Hypenia samples: $\mathrm{Hcal}=H$. calycina; Hcha $=H$. chapadensis $;$ Hine $=H$. inelegans $;$ Hirr $=H$. irregularis $;$ Hjor $=H$. jorgeana $;$ Hmac $=H$. macrantha $;$ Hpar $=H$. paradisi Hpru $=H$. pruinosa $;$ Hpuc $=H$. puchra $;$ Hrup $=H$. rupestre.

to carbon skeletons. Cluster II was clustered and cluster I was mainly split based on samples further displaced to the right of the PC-1 axis (Hrup and Hcal2), which showed a high (low) content of germacrane (bicyclogermacrane) (Figures S1 and S2 in the SI section). The analysis of variance (ANOVA) showed that cluster I revealed significant amounts of caryophyllanes $(19.3 \pm 8.14 \%)$ and aromadendranes $(21.7 \pm 16.3 \%)$, including tricyclane, isolongifolane and italicane as minor constituents $(<1 \%)$. On the other hand, cluster II revealed cadinanes $(45.1 \pm 16.9 \%)$ as the main biosynthetic class, but with the unique occurrence of sesquisabinane, farnesane, indipane, bergamotane, cedrane, thujane and longifolane as minor constituents $(<1.4 \%)$. Percentages of oil constituents (or of the constituents that were rearranged according to carbon skeletons) in clustered samples are shown in the SI section (Tables S2 and S3).

Canonical discriminant analysis (CDA) on chemical data confirmed a priori clusters. An axial representation of CDA results discriminated the two groups based only on the contents of $\alpha$-cadinol and caryophyllene oxide as predictor variables. The fitted model showed high canonical correlation $(R=0.897)$ and significant Wilks' lambda $(0.195$; $p<0.0001$ ), which accounts for a multivariate measure of group differences over both variables. ${ }^{18}$ The discriminant function explained the overall variability $(F=22.74$; degree of freedom, $D F=2$ and $11 ; p<0.0001)$ and distinguished cluster I from II due to high positive and negative scores of caryophyllene oxide and $\alpha$-cadinol, respectively. It was also possible to make an accurate prediction of ca. $94 \%$ well-classification in the original clusters via the cross-validation approach. ${ }^{18}$ This shows that the entire group contributes to discrimination, not only individual plants. The only predicted mismatched classification referred to a sample originating from cluster II (Hcha) which had been classified as belonging to cluster I. Such misclassification might have been caused by a lower level of $\alpha$-cadinol in the sample, which is characteristic of cluster I.

In terms of individual constituents or carbon skeleton contributions to the taxonomic profile, the finding according to which major terpenes such as caryophyllanes/ aromadendranes and cadinanes significantly contributed to separate clusters I and II, respectively, is of particular interest. In fact, flowering Hypenia species from the section Densiflorae were distinguished based on cadinane skeleton, whereas germacranes/bicyclogermacranes separate Laxiflorae flowering individuals. ${ }^{12}$ Apparently, germacranes (via bicyclogermacranes) were converted to aromadendranes, present in the vegetative stage as a discriminant biosynthetic group. Thus, cadinanes such as $\alpha$-muurolol and $\alpha$-cadinol could be chemotaxonomic markers in Densiflorae species regardless of vegetative or flowering stages, whereas germacranes/bicyclogermacranes (e.g. germacrene D and bicyclogermacrane) or aromadendrane biosynthetic pathway (e.g. spathulenol) could be chemotaxonomic markers in Laxiflorae species in flowering or vegetative phenophases, respectively.

Terpenes were described as chemomarkers in other genera, ${ }^{19}$ such as Helichrysum (Asteraceae) and Curcuma (Zingiberaceae) and were proven to be particularly helpful in assessing the taxonomic relationships of several Lamiaceae genera. ${ }^{20}$ In addition, qualitative and quantitative variations in essential oils during plant developmental stages were reported and chemovariations showed ecological and taxonomical significances. ${ }^{21}$

These results support the current division of Hypenia into two taxonomic sections due to remarkable differences in essential oil compositions. Differences in essential oil constituents among Hypenia sections during the developmental stages may be useful for understanding phylogenetic relationships, especially in view of the fact that its species are not easily identified and are not always found during flowering in wild populations. Moreover, the results suggest that the delimitation of $H$. irregularis in the section Densiflorae is more appropriate if applied to the section Laxiflorae. In fact, preliminary results involving a new taxonomic investigation of this species confirm the amendment from the original taxonomic section.

\section{Conclusions}

Essential oil analyses of ten Hypenia species found in Central Brazilian Cerrado have revealed high chemical 
polymorphism, possibly related to genetic influences. Furthermore, the two clustered samples based on multivariate analyses of essential oil chemovariations support the division of species into two taxonomic sections. Cadinane and caryophyllanes/germacranes/ aromadendranes seem to be chemomarkers of sections Densiflorae and Laxiflorae, respectively.

\section{Experimental}

\section{Plant material}

Hypenia samples were collected in the vegetative stage between November 2010 and December 2011 in Goiás State, Brazil (Table S1 in the SI section). Three species were collected from different sampling sites in order to investigate the edaphic influence on oil compositions. The same sampled populations in the flowering stage were identified by one of the authors (M. T. F.) and by Raymond M. Harley, PhD, from the Royal Botanic Gardens, Kew. Vouchers were deposited at the Herbarium of Universidade Federal de Goiás (UFG), Goiás State, Brazil.

\section{Essential oil extraction}

To assess essential oils, 3-5 individuals of each species which originated from 2-3 local populations were pooled and dried at room temperature for seven days at $30{ }^{\circ} \mathrm{C}$ until constant weight. After powdering, the dried phytomass (5-30 g) of each sample was submitted to hydrodistillation $(2 \mathrm{~h})$ using a modified Clevengertype apparatus. At the end of each distillation, the oils were collected and dried with anhydrous $\mathrm{Na}_{2} \mathrm{SO}_{4}$, then transferred to glass flasks where they were kept at a temperature of $-18{ }^{\circ} \mathrm{C}$. Oil yields (\%) were based on the dried weight of plant samples.

\section{Soil analyses}

Soil samples were collected at a $20 \mathrm{~cm}$ depth in all sampling sites and around each population. Then, they were pooled together to form a composite sample for each site. After that, they were air-dried, thoroughly mixed, and sieved $(2 \mathrm{~mm})$. The portion finer than $2 \mathrm{~mm}$ was kept for physical and chemical analyses. The $\mathrm{pH}$ value was determined in a $1: 1 \mathrm{soil} /$ water volume ratio. The cations $\mathrm{Ca}^{2+}, \mathrm{Mg}^{2+}$ and $\mathrm{Al}^{3+}$ were extracted with $1 \mathrm{~mol} \mathrm{~L}^{-1} \mathrm{KCl}$, whereas $\mathrm{P}, \mathrm{K}^{+}, \mathrm{Zn}^{2+}, \mathrm{Cu}^{2+}, \mathrm{Fe}^{3+}$ and $\mathrm{Mn}^{2+}$ were extracted with Mehlich solution. Organic matter, cationic exchange capacity (CEC), potential acidity $\left(\mathrm{H}+\mathrm{Al}^{3+}\right)$ and soil texture were determined by the usual methods. ${ }^{22}$ Soil parameters were ordered in an environmental data matrix with 16 variables for each sampling site. Organic matter, soil textures and other variables were arcsine and $\log (x+1)$-transformed, respectively.

\section{Essential oil analyses}

Oil sample analyses were performed on a gas chromatography-mass spectrometry (GCMS) Shimadzu QP5050A instrument under the following conditions: (i) a CBP-5 (Shimadzu) fused silica capillary column (30 $\mathrm{m} \times 0.25 \mathrm{~mm}$ i.d., $0.25 \mu \mathrm{m}$ film thickness) connected to a quadrupole detector operating in the EI mode at $70 \mathrm{eV}$ with a scan mass range of $40-400 \mathrm{~m} / \mathrm{z}$ at a sampling rate of $1.0 \mathrm{scan} \mathrm{s}^{-1}$; (ii) carrier gas: $\mathrm{He}\left(1 \mathrm{~mL} \mathrm{~min}{ }^{-1}\right)$; (iii) injector and interface temperatures of 220 and $240^{\circ} \mathrm{C}$, respectively, with a split ratio of 1:20. The injection volume was $0.4 \mu \mathrm{L}$ (20\% in hexane) and the oven temperature was raised from 60 to $246{ }^{\circ} \mathrm{C}$ with an increase of $3{ }^{\circ} \mathrm{C} \mathrm{min}^{-1}$, then of $10^{\circ} \mathrm{C} \mathrm{min}^{-1}$ to $270^{\circ} \mathrm{C}$, holding the final temperature for $5 \mathrm{~min}$.

Essential oil constituents were identified by comparing their mass spectra with those from the National Institute of Standards and Technology (NIST), ${ }^{23}$ and by comparing mass spectra and calculated linear retention indices (RI) with values found in the literature, ${ }^{24}$ standards, commercial ylang-ylang (Cananga odorata (Lam.) Hook. F. \& Thoms., Annonaceae), sage clary (Salvia sclarea L., Lamiaceae) and tea-tree (Melaleuca alternifolia Cheel, Myrtaceae) essential oils. Retention indices were obtained by co-injection with a mixture of linear hydrocarbons, $\mathrm{C}_{8}-\mathrm{C}_{32}$ (Sigma, USA), and by the equation of Dool and Kratz. ${ }^{25}$ Total ion chromatograms (TIC) of Hypenia essential oils, standards and commercial essential oils are shown in the SI section (Figures S3 to S20).

\section{Statistical analyses}

PCA and cluster (HCA) analyses using SPAD data mining ${ }^{26}$ were applied to assess the interrelationships between taxa and essential oil constituents. Nearest neighbor complete linkage technique by Benzécri algorithm was used as an index of similarity, ${ }^{27}$ and hierarchical clustering was performed according to Ward's variance minimizing method. ${ }^{28}$ Oil constituents with $\geq 0.5 \%$ arbitrary amounts to the chemical profiles (average values) were initially kept in the original matrix. For variable selection, the threshold of residual eigenvalues $(\leq 0.70)$ in the data matrix was used to establish the maximum number of variables that could be removed. The variables which were effectively eliminated 
revealed the highest loadings in residual eigenvalues. ${ }^{18}$ Prior to the multivariate analysis, the final oil data matrix (14 samples $\times 28$ variables $=392$ pieces of data $)$ was processed by mean centering and auto-scaling.

RDA was applied to describe the patterns of the only explained variation of interrelationships between essential oil constituents, including biosynthetic class and oil yields $(14 \times 92=1288)$, in addition to the interspecific variations as a function of soil parameters $(14 \times 16=224)$, treated as environmental variables. ${ }^{16}$ An unrestricted Monte-Carlo permutation test ( 999 permutations) was used to test the eigenvalue significance of the first canonical axis as well as the sum (trace) of all canonical axes. RDA was performed in CANOCO for Windows, ${ }^{29}$ and oil constituents were arcsine-transformed. Discriminant analysis using SAS CANDISC and SAS DISCRIM procedures were used to distinguish taxa and clusters on the basis of essential oil composition..$^{30}$ The predictive ability of canonical discriminant function was assessed by a cross-validation approach as implemented in SAS.

Multiple comparisons were established by ANOVA using SAS general linear model (GLM) procedure. All data were checked for homoscedasticity with the use of Hartley's test. Whenever heteroscedasticity was observed, the variable was angular or rank-transformed. In addition, whenever a difference in ANOVA was established, a Tukey's post-hoc test was performed. Results are shown by mean values and are joined by the standard deviation of independent measurements. $p$-Values below 0.05 were regarded as significant.

\section{Supplementary Information}

Supplementary data (Tables S1-S3, Figures S1-S20) are available free of charge at http://jbcs.sbq.org.br as a PDF file.

\section{Acknowledgements}

We wish to express our thanks to Dr Raymond M. Harley for kindly assisting us with the botanical identification, and to Dr Anita J. Marsaioli (IQ-Unicamp) for supplying the standards. We are also indebted to Conselho Nacional de Desenvolvimento Científico e Tecnológico (CNPq), Programa de Apoio ao Desenvolvimento Científico e Tecnológico (PADCT III) and Fundação de Apoio à Pesquisa (FUNAPE/UFG) for their financial support, as well as to Conselho de Aperfeiçoamento do Ensino Superior (CAPES) and Fundação de Amparo à Pesquisa do Estado de Goiás (FAPEG) for granting fellowships to M. T. F., D. P. C. and E. C. V.

\section{References}

1. Harley, R. M.; Atkins, S.; Budantsev, A. L.; Cantino, P. D.; Conn, B. J.; Grayer, R.; Harley, M. M.; De Kok, R.; Krestovskaja, T.; Morales, R.; Paton, A. J.; Ryding, O.; Upson, T. In The Families and Genera of Vascular Plants, vol. 7; Kubitzki, K.; Kadereit, J. W., eds.; Springer-Verlag: Berlin, Germany, 2004, chapter 11 .

2. Giulietti, A. M.; Harley, R. M.; Queiroz, L. P.; Wanderley, M. G. L.; Van Den Berg, C.; Conserv. Biol. 2005, 19, 632.

3. Pastori, J. F. B.; Harley, R. M.; Forest, F.; Paton, A.; Van Den Berg, C.; Taxon 2011, 60, 1317.

4. Harley, R. M.; Bot. J. Linn. Soc. 1988, 98, 87.

5. Harley, R. M.; Reynolds, T.; Advances in Labiatae Science, vol. 98; The Royal Botanic Gardens: Kiew, UK, 1992.

6. Epling, C.; Rev. Museo La Plata, Sección Botânica 1949, 30, 153.

7. Briquet, J.; Nat. Pflanzenfam. IV 1897, 3, 183.

8. Martínez-Gordillo, M.; Valencia-Ávalos, S.; Brittonia 2009, 61, 67.

9. Agra, M. F.; Baracho, G. S.; Nurit, K.; Basílio, I. J. L. D.; Coelho, V. P. M.; J. Ethnopharmacol. 2007, 111, 383.

10. David, J. P.; Meira, M.; David, J. M.; Brandão, H. N.; Branco, A.; Agra, M. F.; Barbosa, M. R. V.; Queiroz, L. P.; Giulietti, A. M.; Fitoterapia 2007, 78, 215.

11. Harley, R. M.; Kew Bull. 1974, 29, 125.

12. Silva, J. G.; Faria, M. T.; Oliveira, E. R.; Rezende, M. H.; Ribeiro, D. G.; Ferreira, H. D.; Santos, S. C.; Seraphin, J. C.; Ferri, P. H.; J. Braz. Chem. Soc. 2011, 22, 955.

13. Harley, R. M.; França, F. In Plantas Raras do Brasil; Orgs. Giulietti, A. M.; Rapini,A.; Andrade, M. J. G.; Queiroz, L. P.; Silva, J. M. C., eds.; Conservação Internacional/Universidade Estadual de Feira de Santana: Belo Horizonte, MG, Brasil, 2009, avaiable at http://www.plantasraras.org.br/files/plantas_raras_do_brasil. pdf; Scarano, F. R.; Martinelli, G.; Braz. J. Nat. Conservat. 2010, 8,13 .

14. Dantas, V. L., Batalha, M. A.; Flora 2011, 206, 341.

15. Love, A.; Naik, D.; Basak, S. K.; Babu, S.; Pathak, N.; Babu, C. R.; Chem. Biodiversity 2009, 6, 2263.

16. Lepš, J.; Šmilauer, P.; Multivariate Analysis of Ecological Data Using CANOCO; Cambridge University Press: Cambridge, UK, 2007.

17. Nogueira, T.; Marcelo-Curto, M. J.; Figueiredo, A. C.; Barroso, J. G.; Pedro, L. G.; Rubiolo, P.; Bicchi, P.; Biochem. Syst. Ecol. 2008, 36, 40 .

18. Mardia, K. V.; Kent, J. T.; Bibby, J. M.; Multivariate Analysis; Academic Press: London, UK, 1980.

19. Morone-Fortunato, I.; Montemurro, C.; Ruta, C.; Perrini, R.; Sabetta, W.; Blanco, A.; Lorusso, E.; Avato, P.; Ind. Crop. Prod. 2010, 32, 639; Xiang, Z; Wang, X.; Cai, X.; Zeng, S.; Phytochem. Anal. 2011, 22, 411. 
20. Sanz, J.; Mus, M.; Rosselló, J.; Bot. J. Linn. Soc. 2000, 132, 253; Skaltza, H. D.; Mavrommati, A.; Constantinidis, T.; Phytochemistry 2001, 57, 235; Mohan, L.; Pant, C. C.; Melkani, A. B.; Dev, V.; Nat. Prod. Commun. 2010, 5, 939.

21. Razavi, S. M.; Nejad-Ebrahimi, S.; Nat. Prod. Res. 2011, 25, 627.

22. Silva, S. C.; Manual de Análises Químicas de Solos, Plantas e Fertilizantes, $1^{\text {a }}$ ed.; Embrapa: Brasília, Brasil, 1999.

23. National Institute of Standards and Technology (NIST); PC version of the NIST/EPA/NIH Mass Spectral Database; U.S. Department of Commerce: Gaithersburg, MD, USA, 1998.

24. Adams, R. P.; Identification of Essential Oil Components by Gas Chromatography/Mass Spectrometry, $4^{\text {th }}$ ed.; Allured Publishing Corporation: Illinois, USA, 2007.

25. Dool, H. V. D.; Kratz, P. D.; J. Chromatogr. 1963, 11, 463.
26. Système Portable pour l'Analyse des Données (SPAD); Coheris Corp.: Suresnes, France, 2002.

27. Benzécri, J. P.; L'Analyse des Données: la Taxinomie, Tome 1; Dunod: Paris, France, 1980.

28. Ward, J. H.; J. Am. Stat. Assoc. 1963, 58, 238.

29. Ter Braak, C. J. F.; Šmilauer, P.; CANOCO Reference Manual and CanoDraw for Windows User's Guide: Software for Canonical Community Ordination, version 4.5; Microcomputer Power: New York, NY, USA, 2002.

30. Statistical Analysis System; SAS Institute Inc.: Cary, NC, USA, 1996.

Submitted: April 19, 2012

Published online: October 11, 2012 